\title{
Photogrammetric Measurement of the Classic Marathon Course
}

Ino Papageorgaki, Sevi Tapinaki, Andreas Georgopoulos, and Charalambos Ioannidis

\author{
Laboratory of Photogrammetry, National Technical University of Athens, Greece \\ inw20@hotmail.com, sevi@survey.ntua.gr, drag@central.ntua.gr, \\ cioannidasurvey.ntua.gr
}

\begin{abstract}
In this paper an alternative method for measuring the Classic Marathon Course using photogrammetric methodology is presented and examined. The course is surveyed in stereoscopic environment and therefore the measurement is performed directly in $3 \mathrm{D}$ space. The produced elevation data after the processing procedure are presented in the section plan at a scale of 1:5.000. The Digital Surface Model, with a step of $5 \mathrm{~m}$, and the orthophotomap at a scale 1:2.000 of a corridor area, with width of $100 \mathrm{~m}$ on either side of the course represents an additional product of the photogrammetric method. At the end, in this thesis the photogrammetric methodology is being assessed and evaluated, but also it is being compared with the conventional method of the calibrated bicycle and finally are conclusions drawn and proposals are attempted.
\end{abstract}

Keywords: Orthophotography, Photogrammetric Measurement, GPS, Cultural Heritage.

\section{Introduction}

One of the more important events of modern Olympic Games is the Marathon Run, which is run on a course, usually outside the main stadium, with a total length of $42.195 \mathrm{~m}$. This event was included in the Olympic program since the first modern Olympics in 1896 in recollection of the heroic achievement of the warrior - messenger who first announced to the Athenians the victory of the Greeks in the battle of Marathon in 490 B.C. The Classic Marathon Course (Fig. 1) is the route from Marathon, Attica up to the Panathinaikon stadium (Fig. 2) and it measures $42.195 \mathrm{~m}$. This is also the distance that marathon runners cover in the event of the Marathon Run in all modern Olympic Games, but also in all other national or international Marathon Runs. Although the marathon run was one of the first modern Olympic Games events its length was not strictly determined until 1924. In 1896, Spyros Louis, the first Gold medal winner ran 40.000m (http://www.sport.gr/default.asp?pid=52\&aid=51139). Ten years later, the runners who took part in the marathon run of the "Half" Olympiad) in Athens in 1906, ran 41.860m. In 1908, in the Olympic Games of London the length changed to $42.195 \mathrm{~m}$ so that the runners would pass in front of the Royal Palaces. It took another 16 years before the differentiations of the distance of the marathon run stopped. The length was officially decided to be $42.195 \mathrm{~m}$ in the Olympic Games of Paris in 1924 (http://www.sport.gr/default.asp?pid=52\&aid=17305). 


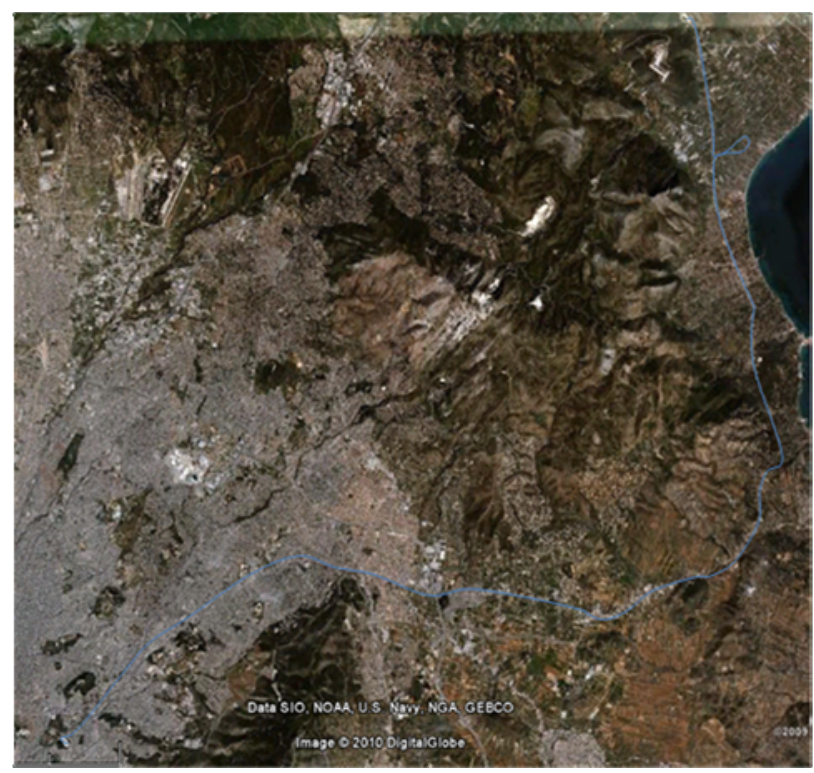

Fig. 1. The Classic Marathon Course

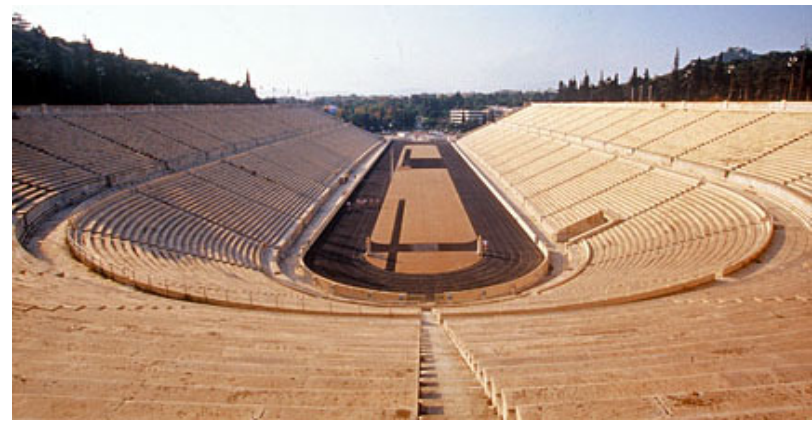

Fig. 2. The Panathinaikon Satdium (Kallimarmaro) in Athens

For the validation of a record time, the length of a course must be measured with certain accuracy. This of course is the case not only for Marathon runs, but for all other events, be it inside or outside the track of a stadium. Over the time widely accepted rules have been developed, in order to ensure the validation both of the organized event and the record times of the winners. As of 1.1.2004 a commonly accepted regulation for the road race courses out of stadium is in effect, compiled by the IAAF (World Federation of Track and Field) and the AIMS (World Union of of Street Runs). This regulation, among other issues, dictates how a street course should be measured, in order to ensure that the athletes will run at least the predetermined length. 
Over the years several methods for measuring street course have been proposed, tested and applied. Topographic methods, using all sorts of measuring techniques (staves, steel tapes, EDM's etc) have been used in the Seoul Olympics (Seoul Olympic Organizing Committee, 1988). Other efforts include the application of a DGPS system (Gordon, 2005), the use of a terrestrial Laser scanner (Tsakiri et al, 2004), measuring the distance on a map and odometers adapted on cars. However the only method nowadays recognized as valid by the IAAF/AIMS (IAAF, 2004) for measuring a street course, is that of the calibrated bicycle.

In the present paper an alternative method for measuring the length of the Classic Marathon Course using photogrammetric methodology is described and assessed. The basic argument for using photogrammetry in the measurement of a Marathon Course is based on the fact that the photogrammetric procedure for length measurement can be as effective as the conventional method of the calibrated bicycle. However, careful selection of the suitable photogrammetric method should be carried out, in order to achieve the required result, mainly in terms of accuracy and cost. Photogrammetric methods are actually non-contact methods and in this way tedious fieldwork may be minimized.

\section{The Method of the Calibrated Bicycle}

\subsection{Requirements and Specifications of IAAF/AIMS}

Every measurement technique proposed for measuring road courses should comply by the requirements and specifications set by IAAF, independently of the methodology employed.

These specifications are briefly as follows:

- The distance of the Marathon Course should be between $42.195 \mathrm{~m}$ and $42.237 \mathrm{~m}$, that is $+0.1 \%$ overshooting from the usual distance and only additive.

- The distance should be measured with precision better than a part in the 1000 $(0.1 \%)$. This means that the whole distance should be measures with an ambiguity of $42 \mathrm{~m}$ over the whole length.

- The measured course should follow the ideal path which the athletes will decide to run or walk.

- If the calibrated bicycle method is to be used, the bicycles should be calibrated before and after the process of measurement.

- The process of measurement should be applied twice and the results of these two independent measurements should not differ more than $0.8 \%$. Otherwise a third measurement should be carried out.

- The shortest possible route is defined as the one which runs $0.30 \mathrm{~m}$ away from the curb, or the end of road surface when a curb does not exist. If constructions are present at the side of the road, such as walls, the shortest possible route should be considered a path $0.61 \mathrm{~m}$ away from them. 


\subsection{Analysis of the Conventional Method}

The basic idea of the conventional method of measurement is to determine the ratio of the course length covered by a complete revolution of the bicycle wheel and then to count the number of revolutions necessary to cover the whole course. The calibration of this system consists of determining the "bicycle constant" by determining the number of wheel revolutions needed to cover a length known with sufficient accuracy. The method is simple and direct, but a lot of important details are involved and certain steps should be followed so that it results to an acceptable and complete measurement of a course (IAAF, 2004).

For the determination of the length of the Marathon course the line of departure, the finishing line and the shortest possible route should be first of all established and fixed. The final product with the results of the described processes is a document which includes a map of the entire marathon course and all characteristics of the route.

For the choice and the measurement of the calibration length several considerations should be taken into account. Firstly a straight and level part of a paved road should be chosen as the calibration field. It should be approximately $300-500 \mathrm{~m}$ in length and it is desired not to have any parked vehicles. Secondly, the length of the calibration field should be accurately measured using a steel tape with maximum length of $30 \mathrm{~m}$, with practically no change of length at $20^{\circ} \mathrm{C}$. The measurement should be executed twice and if the difference is more than $0.8 \%$, a third measurement is repeated. The bicycle used for the course measurement is fitted with a special device called Jones counter (Fig. 3). It actually converts the wheel revolutions to a five or six digit number, which, after careful calibration, provides the bicycle constant. For the calibration of the bicycle the cyclist should cover the calibration field, four times before and four times after the measurement, in both directions.

The bicycle constant is the mean of the four values from the four calibration runs, divided by the calibration distance (in $\mathrm{km}$ ). The result is the number of units of Jones

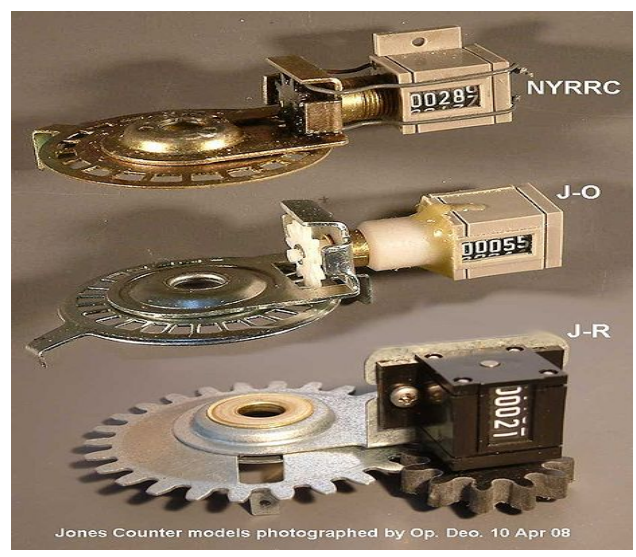

Fig. 3. Various types of Jones Counters 
Counter per kilometer, which is further multiplied by 1.001, the factor SCPV (short course prevention value), in order to ensure that the final course measurement result is definitely not less (by 0.001 ) than the desired distance. This results to a total distance for the Marathon course between the values $42.195 \mathrm{~m} \mathrm{42.237m}$. In this way, runners are prevented from covering a distance shorter than $42.195 \mathrm{~m}$.

The actual measurement of the road race course is determined by the measurement of the shortest possible route (SPR). The shortest possible route is theoretically determined with clarity and precision following the IAAF/AIMS specifications. The determination of road race course in this way ensures that all runners will run at least the predefined distance of the road race course. The shortest possible route in various road configurations is presented Fig. 4.

After the actual course length measurement, the bicycle calibration process is repeated. The objective of this re-calibration after the measurement is to make sure that no change in the bicycle constant has occurred. The re-calibration of the bicycle is essential to be carried out immediately after the actual measurements.
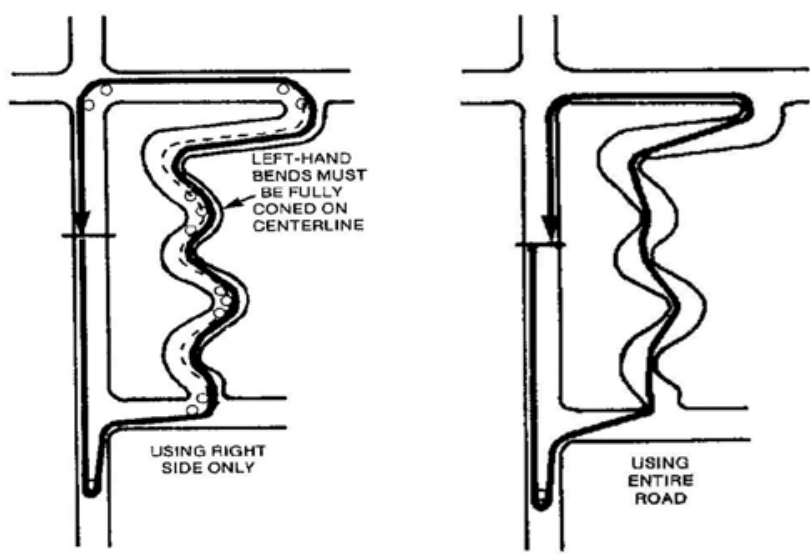

Fig. 4. The SPR in various cases

To calculate the length of the road race course, the Constant for the Day must be determined first. This is the average of the two values resulting from the two calibrations. Then the actual course is run by the bicycle, following of course the shortest possible route, and the total number of counts is recorded. This value is then divided by the constant for the day. The result obtained is the length of the road race course. If the measurements last more than one day, the final lengths of each day are added. The measurement process described above should be applied twice, where the second time serves for checking. In the event that a few meters are either surplus or missing from the final result, adequate adaptations are made using simple steel tape measurements, usually by appropriately shifting the start or finishing line. The whole measurement process, as described above, and for it to be officially recognized, should be properly documented (Fig. 5). The documentation of the measurement involves the production of a map of the course, which contains all necessary details used for the measurement process. 


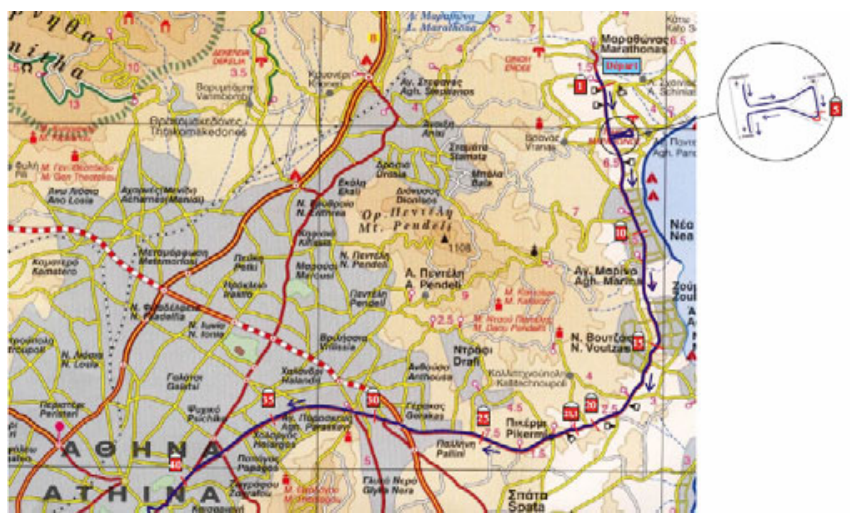

Fig. 5. General map of the Classic Marathon Course measurement documentation

The documentation must be sufficient to allow the course to be checked if a remeasurement is for any reason required, e.g. after a world best performance has been recorded. In the documentation folder all necessary details of the road race course should be included, which are adequate for the race director to re-establish the course even after major construction works on the roads.

\section{Photogrammetric Methodology}

Photogrammetry is the art, science and technique for the acquisition of reliable metric information on natural objects and the environment, via processes of recording, measuring and interpreting, photographic images, electromagnetic radiation recordings and other phenomena (McGlone, 2004). The basic idea for the use of photogrammetry in the measurement of the Marathon course is based on the fact that photogrammetric measurements could perhaps be as effective as the conventional method of the calibrated bicycle, which is normally used for the measurement of road race courses. It is proposed that photogrammetric methodology should be used to enhance, and not necessarily to replace, the existing methods, especially in cases of long distances as a Marathon Course.

Before the application of any photogrammetric methodology, several considerations are necessary, concerning the data characteristics and the various parameters of the methods that will be used in order to achieve the required objective. The small scale of aerial images used by Photogrammetry, enables the measurement of the whole course, contrary to the conventional surveying methods, i.e. use of total stations or terrestrial scanners, which are unable to cover of whole object at once. Although photogrammetry may be somewhat dependent on topographic measurements, the length of an object may be determined either stereoscopically, by directly measuring in the 3D model, or monoscopically, by measuring on an orthogonal projection (i.e. orthophotograph) and indirectly taking into account the elevation via the existing DTM (Fig. 6). An orthophotograph is a photographic picture that has been reduced differentially to an orthogonal projection, which has no perspective deformations. 
Its main advantage is the standard scale, similar to a conventional map, it contains however all qualitative information of a photographic image. For the production of an orthophotograph it is necessary to have oriented images and a sufficient description of the relief.

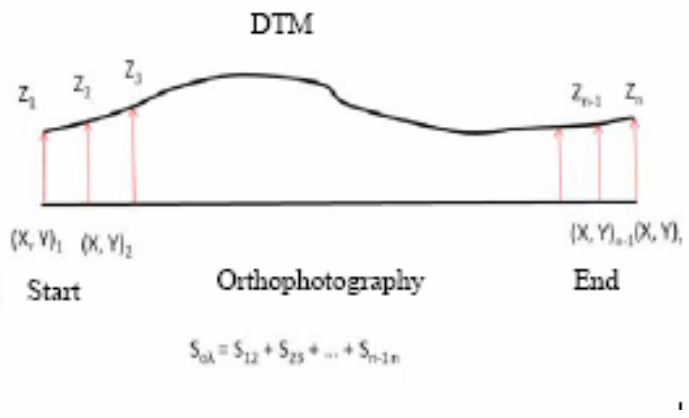

Fig. 6. Monoscopic measurement of course length

Finally the calculation of the length is given by the equation:

$$
\mathrm{D}=\sqrt{\Delta X^{2}+\Delta Y^{2}+\Delta Z^{2}}
$$

Where $\mathrm{X}, \mathrm{Y}, \mathrm{Z}$ are the three-dimensional coordinates of the vertices.

\section{Implementation and Results}

The proposed photogrammetric methodology for the measurement of Classic Marathon Course includes five main stages:

Determination, acquisition and preparation of the necessary data: First of all aerial photographs of suitable scale are required, imaging the whole course. A total of 34 aerial photographs (diapositives) of scale 1:15.000 and taken in 2005 were obtained from the Hellenic Cadastral and Mapping Organization. The aerial camera used was ZEISS RMK TOP 15 with focal length $153,120 \mathrm{~mm}$, format $230 \times 230 \mathrm{~mm}$ and eight fiducial marks (Fig. 7). The available scale of aerial photographs was considered suitable in order to ensure the specified accuracy for the measurement of Classic Marathon Course. The initial coloured aerial photographs were scanned with Photoscan PS1 by Z/I, a digital photogrammetric scanner of large accuracy, with spatial resolution, i.e. pixel size, $21 \mu \mathrm{m}$. In this way the recognition of objects larger than $0,15 \mathrm{~m}$ on the digital images is possible.

Photogrammetric processing: All necessary photogrammetric processing, such as interior and exterior orientation, stereoscopic observations and plotting, were carried out with the digital photogrammetric station PHOTOMOD v.4.4 by Racurs. All 34 images with $60 \%$ overlap were imported in the digital photogrammetric workstation 


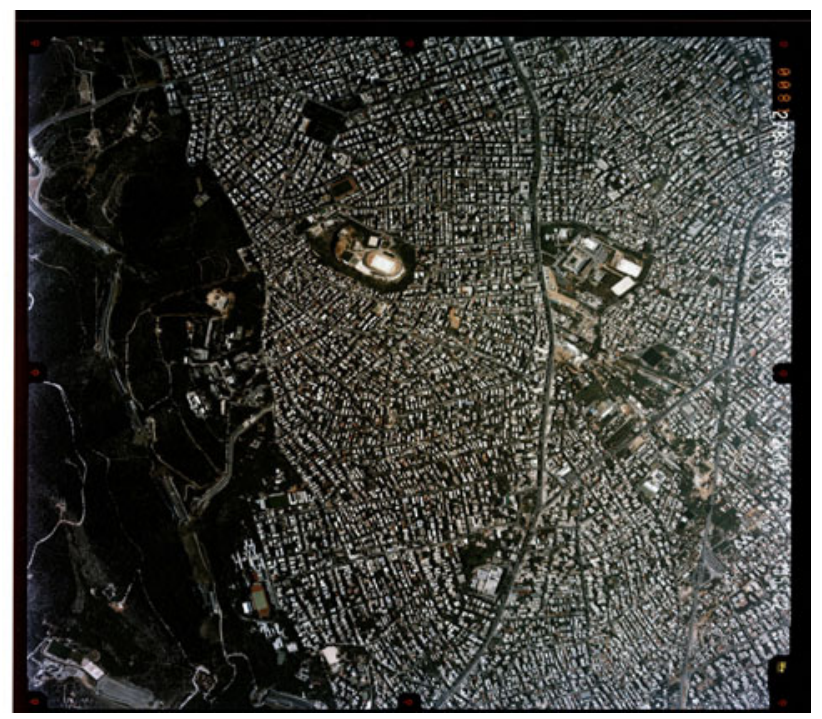

Fig. 7. One of the coloured aerial photographs

and created a block of twelve strips (ten strips of two images each, one of nine and one of six images) with $30 \%$ side overlap. The parameters of the interior orientation were given by the calibration report accompanying the aerial photographs.

For the exterior orientation of the images a standard bundle adjustment was applied. For this process control points are essential, as they are necessary to connect the block of images with the geodetic reference system. Control points materialize the reference system of the object and contribute to the absolute orientation of models. The position of the control points influences considerably the course of photogrammetric processing as well as the quality of the final products. The final positions of the control points were determined with stereoscopic observation of the stereo models in order to fulfil the following conditions:

- Uniform distribution of control points, so that control points are situated along the perimeter of the region of interest and to establish strong geometry of the system.

- Ensure that control points exist at least every five stereo models (approximately $10 \mathrm{~km})$,

- Control points should be visible and recognizable on the images,

- Ensure high contrast between the point and the surrounding environment,

- If control points are visible in more than two images, economy is accomplished,

- Control points must be in the overlapping part of the images

- Control points should be easily visited and ensure open horizon vision, because it is essential that no obstacles exist (buildings, bridges, trees, antennas etc.) that would interrupt the reception of the satellite signal during the GPS measurements. 
The measurement and determination of geodetic coordinates of control points was executed with the use of GPS measurements. 34 points were measured in total, 17 of which were used as control points for the bundle adjustment of the triangulation and the rest were reserved as check points (Fig. 8). Static differential measurements were used and each base less than $8 \mathrm{~km}$ was measured for 20 minutes, in such a way the accuracy of every measurement was estimated at $0,06 \mathrm{~m}$. The block of all images with the distribution of points, after bundle adjustment, is shown in Figure 9.

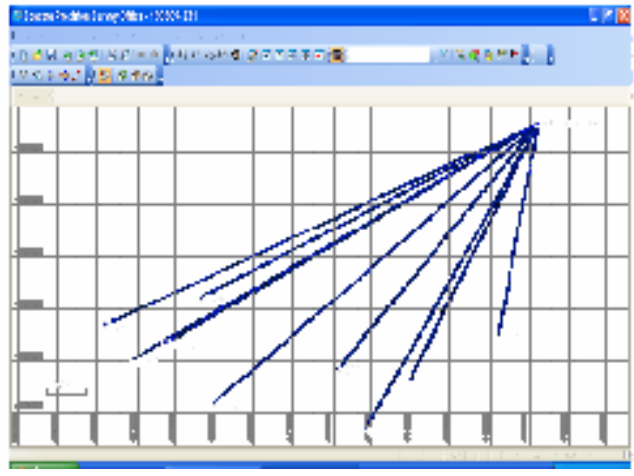

Fig. 8. Screenshot of the computation of several measured bases with GPS

\section{Three-dimensional surveying of Classic Marathon Course using stereoscopic} observation: The whole procedure of mapping the course was carried out using the stereoscopic environment of PHOTOMOD, according to the IAAF/AIMS specifications (IAAF 2004). Utmost attention was paid so that the shortest possible route was followed by the floating mark. Whenever possible large straight lines were used (up to 200m), while in cases where circular arcs were needed, the distance between successive vertices was reduced to approximately $20 \mathrm{~m}$. The result of the above process was a 3D polyline that is composed of individual 3D lines and constitutes the total classic Marathon course, as determined by the operator. Drawing of the shortest possible route is a difficult process for individuals that come in contact with this task for the first time and have no experience in this whatsoever. Hence, the final course mapped was also examined and approved by an IAAF certified race course measurer.

Measurement of the length directly in 3D space: For measuring the mapped course the following procedure was applied. The 3D polyline was exported to $\mathrm{dxf}$ format for the measurement to be carried out in a CAD program. The measurement of the total length of the Classic Marathon Course was carried out within the AutoCAD 3D ${ }^{\circledR}$ environment. Each individual line was measured using the appropriate CAD tools and the total length of the course was calculated by simply adding all these lengths together. The three dimensional line ensures that the measured length corresponds to the real length of the course. 


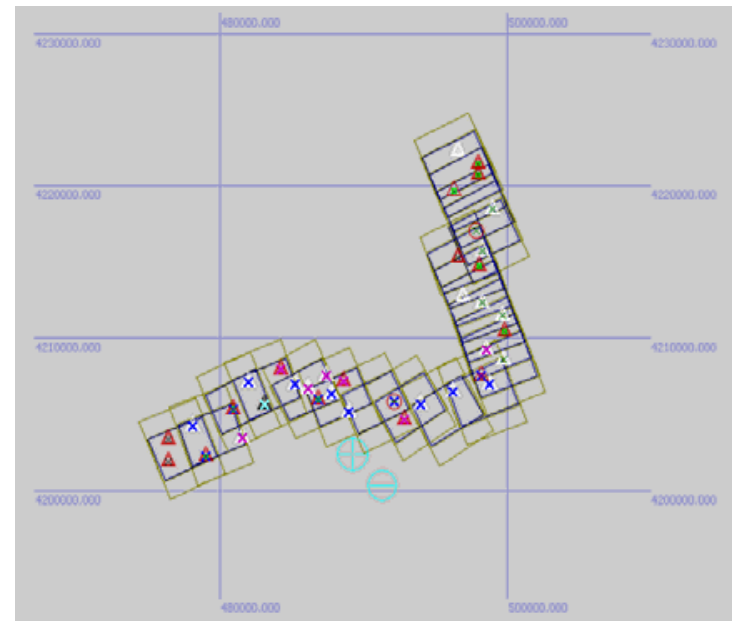

Fig. 9. The block of images with the distribution of points

Evaluation of methodology and results. The photogrammetric methodology is thoroughly evaluated, through the evaluation of the measurement results and the final products as far as effectiveness, accuracy, required time and cost are concerned.

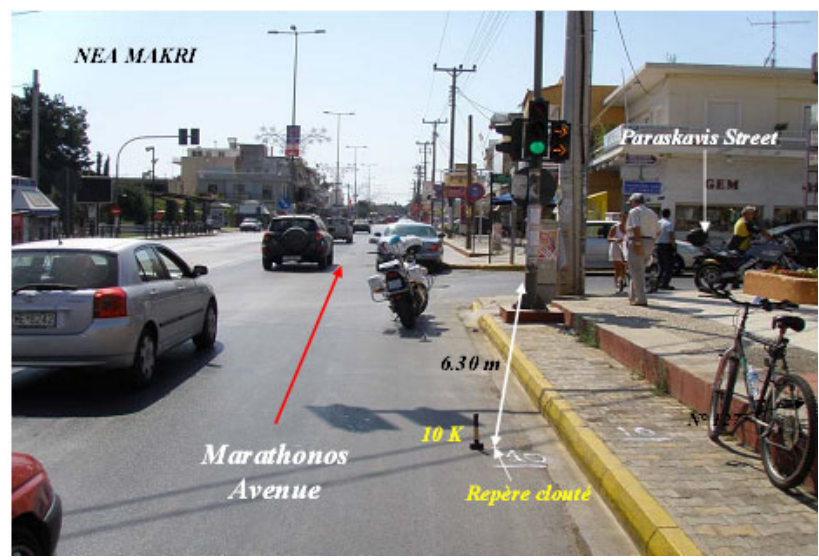

Fig. 10. Documented measurements of the 10 th kilometer. (Grall, 2008)

For this purpose the length resulting from the photogrammetric method was compared with the length between the characteristic points which correspond to each kilometre of the course, as documented in the relevant Documentation Folder. The locations of these points are well known and their exact position is recorded both by coloured photos and with accurate measurements, which took place at the same time as the measurement of course with the calibrated bicycle (Fig. 10). 
Table 1. Distance check between certain kilometres

\begin{tabular}{||c|c||c|c||}
\hline \multicolumn{2}{|c||}{ Calibrated Bicycle } & \multicolumn{2}{c|}{ Photogrammetric Measurement } \\
\hline $\begin{array}{c}\text { Distance from } \\
\text { Start }(\mathbf{k m})\end{array}$ & $\begin{array}{c}\text { Distance between } \\
\mathbf{( m )}\end{array}$ & $\begin{array}{c}\text { Distance between } \\
\mathbf{( m )}\end{array}$ & $\begin{array}{c}\text { Distance from } \\
\text { Start }(\mathbf{m})\end{array}$ \\
\hline 4 & $4.000,00$ & $3.819,83$ & $3.819,83$ \\
\hline 5 & $1.000,00$ & $1.180,43$ & $5.000,27$ \\
\hline 8 & $3.000,00$ & $3.147,70$ & $8.147,96$ \\
\hline 12 & $4.000,00$ & $3.852,11$ & $12.000,07$ \\
\hline 18 & $6.000,00$ & $6.004,02$ & $18.004,09$ \\
\hline 25 & $7.000,00$ & $7.047,24$ & $25.051,33$ \\
\hline 28 & $3.000,00$ & $2.978,14$ & $28.029,46$ \\
\hline 29 & $1.000,00$ & $1.045,56$ & $29.075,02$ \\
\hline 30 & $1.000,00$ & $1.000,90$ & $30.075,92$ \\
\hline 35 & $5.000,00$ & $4.966,95$ & $35.042,87$ \\
\hline 39 & $4.000,00$ & $3.978,39$ & $39.021,27$ \\
\hline $\mathbf{4 2 , 1 9 5} \mathbf{~ k m}$ & $3.195,00$ & $3.208,43$ & $\mathbf{4 2 . 2 2 9 , 6 9}$ \\
\hline
\end{tabular}

\section{Difference between the two methods $=34,69 \mathrm{~m}(0.08 \%)$}

Table 1 shows that, for certain parts of the course, the difference between the distance that was measured with the photogrammetric method and the one that results from the conventional method could be as high as $148 \mathrm{~m}$, but the total difference is only $34,69 \mathrm{~m}$. This large difference occurs between the $5^{\text {th }}$ and the $8^{\text {th }}$ kilometre, where from the fifth up to the sixth kilometre the athletes are obliged to follow a circular course around the Marathon Tomb, commemorating the battle in 460B.C. This is, obviously, due to the fact that the definition of the shortest possible route (SPR) along an arc demands the use of more points (vertices), while for the large straight lines two points are enough, thus ensuring smaller discrepancies. Although, the use of large straight parts ensures more accurate length measurement, part of the elevation information is lost. However, this does not influence the resulting length for small distances.

The average length from vertex to vertex of the 3D polyline, as mapped within the digital photogrammetric workstation environment, is approximately $50 \mathrm{~m}$. The error corresponding to the measurement of each part is estimated to $25 \mathrm{~mm}$. Also, the photogrammetric processing propagates errors to each point determined photogrammetrically. For the determination of the standard deviations $\sigma_{x}, \sigma_{y}, \sigma_{z}$ of the measurements of the coordinates of each vertex of the $3 \mathrm{D}$ polyline, with the photogrammetric process, four check points were measured 20 times each in stereoscopic mode. The average of the residuals of each coordinate from the measured ones with the GPS, determines the requested standard deviations: $\sigma_{\mathrm{x}}= \pm 0,119 \mathrm{~m}, \sigma_{\mathrm{y}}= \pm 0,112 \mathrm{~m} \sigma_{\mathrm{z}}= \pm$ $0,179 \mathrm{~m}$. The mathematical relation which was used for the calculation of the total error of the measured distance $\mathrm{D}$ with vertices of known coordinates $(\mathrm{X}, \mathrm{Y}, \mathrm{Z})$ in the reference system (GGRS' 87) and the error of the determination of each coordinate $\left(\sigma_{\mathrm{xi}}, \sigma_{\mathrm{yi}}, \sigma_{\mathrm{zi}}\right)$ with the above method is: 


$$
\begin{aligned}
& \sigma_{D_{i j}}^{2}=\sigma_{x j}^{2}\left(\frac{x_{j}-x_{i}}{D_{i j}}\right)^{2}+\sigma^{2}{ }_{x i}\left(\frac{x_{j}-x_{i}}{D_{i j}}\right)^{2}+\sigma^{2}{ }_{y j}\left(\frac{y_{j}-y_{i}}{D_{i j}}\right)^{2}+\sigma^{2}{ }_{y i}\left(\frac{y_{j}-y_{i}}{D_{i j}}\right)^{2}+ \\
& \sigma_{z j}^{2}\left(\frac{z_{j}-z_{i}}{D_{i j}}\right)^{2}+\sigma^{2}{ }_{z i}\left(\frac{z_{j}-z_{i}}{D_{i j}}\right)^{2}
\end{aligned}
$$

Where $D=\sqrt{\left(\Delta \mathrm{x}^{2}+\Delta \mathrm{y}^{2}+\Delta \mathrm{H}^{2}\right)}$ and $\sigma_{\mathrm{xi}}=0,119 \mathrm{~m}, \sigma_{\mathrm{yi}}=0,112 \mathrm{~m}, \sigma_{\mathrm{zi}}=0,179 \mathrm{~m}$.

$$
\begin{aligned}
& \sigma_{D}^{2}=\Sigma \sigma_{D_{i j}}^{2} \text { and } \sigma_{D}= \pm \sqrt{\sigma^{2}{ }_{D}} \\
& \sigma_{D}= \pm 4,69 \mathrm{~m}
\end{aligned}
$$

On the whole, the distance was calculated $42.229 \mathrm{~m} \pm 4,69 \mathrm{~m}$. This deviation is better than $0,1 \%$ required by the IAAF regulations $(42.216 \mathrm{~m} \pm 21 \mathrm{~m})$. It is also observed that the larger error is in the determination of the $\mathrm{Z}$ coordinate. This was expected since during stereoscopic observation the step of the floating point movement along $\mathrm{Z}$ axis is approximately $0,15 \mathrm{~m}$, consequently each surface was approached with this uncertainty.

\section{Conclusions}

The recent technological developments have definitely affected all related disciplines. Especially in the case of digital photogrammetry combined with the contemporary aerial photography progress, the ideal conditions have been created for the production of exceptionally highly accurate base maps, or indeed documents, either in vector or in raster format. With the use of photogrammetric methodology the measurement of any size of object is possible. In cases of large areas or objects the application of conventional survey measurement methods is considered inefficient. On the contrary with photogrammetry where the scales of aerial photographs or satellite images are small, without affecting the final accuracy, raster products with metric attributes may be easily produced.

It has been shown that digital photogrammetric methodology may be a sound alternative to classical measuring techniques, as far as road race course measurement tasks is concerned. Firstly in terms of accuracy, the use of relatively small scale (1:15.000) aerial images with a medium scanning resolution $(21 \mu \mathrm{m}$ pixel size $)$ is more than adequate to ensure stereoscopic measurements within the IAAF regulations, both as far as the absolute accuracy of each measurement is concerned, but also for determining the Shortest Possible Route. The use of simple GPS measurements for the control points is fast -only 20 minutes measuring time for each base- accurate -accuracy of a few centimetres is more than enough for such an application- and, of course, it is simpler than any conventional survey technique.

Contemporary Digital Photogrammetric Workstations, as indeed the one used in this project (PHOTOMOD v. 4.4), are generally easy to learn and provide all necessary computational tools for adjusting the photogrammetric networks, thus ensuring the required accuracy and reliability in the results. The conventional method for the Classic Marathon Course measurement requires that the length of the course is never measured less than $42.195 \mathrm{~m}$ and never greater than $42.237 \mathrm{~m}$ or otherwise 
$42.195 \mathrm{~m}+42 \mathrm{~m}$. That could equivalently be expressed as $42.216 \mathrm{~m} \pm 21 \mathrm{~m}$ and in this form it may be compared with the result of the photogrammetric process which is $42.229 \mathrm{~m} \pm 4,7 \mathrm{~m}$. It may be concluded that in terms of accuracy, the result of the photogrammetric methodology, as implemented in this project, presents a small deviation and the final result is acceptable and within the IAAF regulations.

In terms of time and related cost, photogrammetric methodology is definitely more demanding. Usually conventional official measurers work voluntarily, hence their cost is minimal. The full Marathon Race Course measurement procedure lasts for about two days, including bicycle calibration. On the contrary, aerial photo acquisition, preparation for GCPs measurement, GPS measurements, photogrammetric adjustments and stereo mapping take longer and demand more cost. Hence, as set out at the beginning, photogrammetry is not proposed to replace conventional methodology for the task, if the course measurement is needed once.

The benefits, however, are becoming obvious if the course should be measured repeatedly, or if there are slight alterations to the course and a re-measure is desperately needed. Moreover, it is possible to have a 3D visualization of the course, in order to use it for any kind of simulations. The results of this research, such as the three dimensional data of the surroundings of the whole course could be used as the background for the development of a three dimensional system, which will be targeted to athletes and trainers and will provide a 3D tour of the race course and virtual training. The Classic Marathon Course is considered to be a very difficult course because of the elevation differences. With this system the runners participating to the race will have the opportunity to study the course wherever they are. Alternatively, the 3D visualization of the course could be projected with special monitors in front of exercise treadmills. The runners, during training, will watch in real time the landscape variations of the Classic Marathon Course.

\section{Acknowledgements}

We would like to thank Mr. George Doussis, for the very useful information and data he provided, the detailed description of the conventional method of measurement of Marathon courses and the time that he devoted to us. Mr. George Doussis is an active amateur runner and trainer. He participated in the organisation and measurement of several courses during the Olympic Games of Athens, among them the Classic Marathon Course.

\section{References}

1. Doussis, G.: The "unmeasured" courses (May 2007), http: / / www . runningnews.gr / ?id=2821 (accessed April 5, 2007)

2. Free Encyclopedia, http://el.wikipedia.org/wiki/M•-.......\#.CE.99.CF.83 .CF. 84 . CE.BF.CF . 81.CE.AF.CE.B1 (accessed September 11, 2009)

3. Ioannidis, C.: Photogrammetry II. Lecture Notes. National Technical University of Athens, Athens (2006) (in Greek) 
4. Gordon, S.: Course Measurement (February 2005), http: / /www.runscore.com/coursemeasurement/Articles / Spac $\% 2$ Meas.pdf (accessed 06/04/2009)

5. Grall, J.-M.: Dossier de Measurage Athens Classic Marathon (November 2008)

6. IAAF (International Association of Athletics Federations) 2004, The Measurement of RoadRace Courses, second edition (updated 2008)

7. Kraus, K.: Photogrammetry, Technical Chamber of Greece, 2nd edn., Athens, vol. 1, p. 361 (2003) (in Greek) ISBN 960-7018-90-7

8. Racurs, PhotoMod 4.4 User Manual, Moscow, Russia (2007)

9. Seoul Olympic Organizing Committee, Marathon / Race Walks Courses Study and Measurement, Seoul, p. 231 (1998)

10. The variation of Marathon course (27/04/2008), http: / / www. sport.gr/default.asp?pid=52\&aid=17305 (accessed 2/11/2009)

11. The distance of Marathon course (04/11/2009), http: / / www. sport.gr/default.asp?pid=52\&aid=51139 (accessed 05/11/2009)

12. Tsakiri, M., Stathas, D., Arabatzi, O.: Advanced Surveying Techniques for Measuring the Marathon Course. In: Proc. of FIG Working Week 2004, Athens, Greece, May 22-27 (2004) 